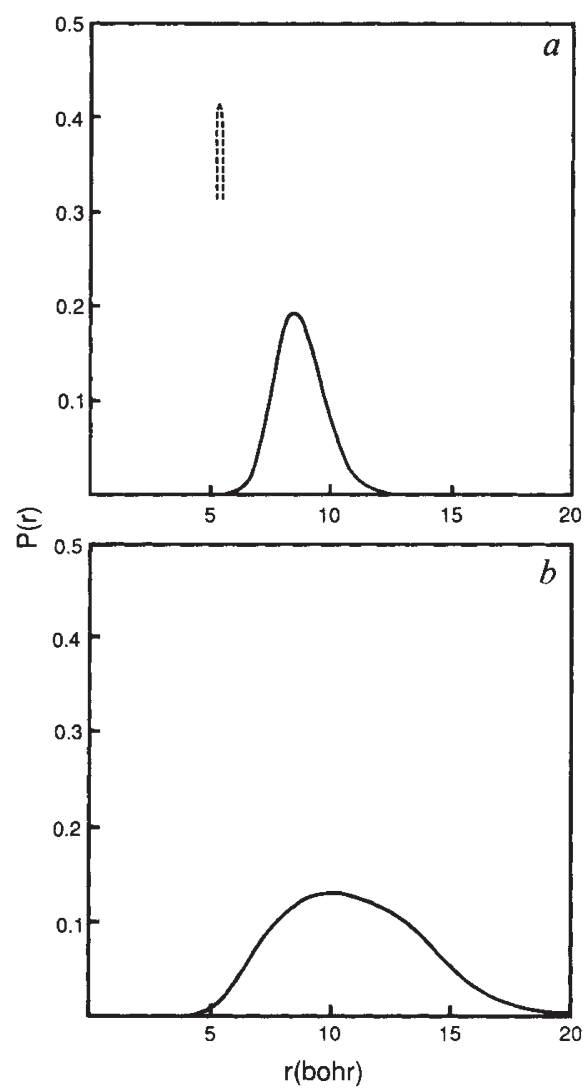

FIG. 2 The interatomic separation distribution for $\mathrm{He}-\mathrm{Xe} \mathrm{(a)} \mathrm{and} \mathrm{He}-\mathrm{He}(b)$ in the ground state of $\mathrm{He}_{2} . \mathrm{Xe}$. The broken line shows the distribution to be expected from a rigid rotor.

ximation). Thus, at temperatures close to absolute zero the relative positions of the atoms in a molecule or complex should be more or less fixed, with only the unavoidable zero-point vibrational motion contributing to any movement. From an analysis of spectra taken under these conditions, an equilibrium structure can be assigned. Earlier studies of $\mathrm{Ne}_{2} \cdot \mathrm{Cl}_{2}$ and $\mathrm{Ar}_{2} \cdot \mathrm{Cl}_{2}$ by Janda and colleagues $^{2,3}$ showed that features in the spectra due to rotational motion could be accounted for by assuming that the clusters behave as rigid rotors. The difference with $\mathrm{He}_{2} \cdot \mathrm{Cl}_{2}$ is that, despite being below a temperature of $1 \mathrm{~K}$, it does not follow the same pattern'. Instead, the spectra show that, relative to the chlorine molecule, the helium atoms move considerably so that it is not possible to assign the complex an equilibrium structure.

The complex has four possible equilibrium configurations (Fig. 1) which can be calculated from a simple consideration of the interaction between the two helium atoms and between the molecule and each helium atom. The energy of each structure lies below the zero-point vibrational energy of the complex, so that even in their lowest energy state, the helium atoms have sufficient mobility to sample each structure on a time- scale of several vibrational periods $\left(10^{-12} \mathrm{~s}\right)$.

Several research groups ${ }^{4,5}$ have attempted to calculate the dynamic properties of these and related systems (such as $\left.\mathrm{He}_{2} . \mathrm{Xe}\right) ; \mathrm{a}$ general conclusion appears to be that the complexes are best described as extremely floppy and liquid-like, and as having structural distributions rather than equilibrium configurations (Fig. 2). The broad spread of $\mathrm{Xe}-\mathrm{He}$ and $\mathrm{He}-\mathrm{He}$ interatomic distances shown in Fig. 2 for $\mathrm{He}_{2}$. Xe is characteristic of large amplitude motion over a very shallow potential-energy surface. A similar result would be expected for the $\mathrm{He}_{2} \cdot \mathrm{Cl}_{2}$ complex as it interconverts between the structures shown in Fig. 1. Taking the concept of a structural distribution one step further, one might liken Fig. 2 to one component of the pair or radial distribution function of a liquid; thus, as far as the spectroscopy of $\mathrm{Cl}_{2} \cdot \mathrm{He}_{2}$ is concerned, the core chlorine molecule is being perturbed by a continual sequence of (elastic) quasicollisions with a liquid-like helium component.

From an experimental viewpoint, adiabatic expansion provides some (loose) control over the final temperature that can be achieved. Therefore, as mixed clusters of this type increase in size, it should be possible to identify either of two limiting physical states from their spectroscopy. With the heavier inert-gas atoms, such as argon and krypton, for which the interatomic interaction is comparatively strong, it seems that trapped molecules have spectra that are similar to those of matrixisolated species ${ }^{6}$. Thus we would conclude that the clusters are solid-like. But by varying the expansion gas pressure, it has been suggested ${ }^{7}$, a phase transition can be promoted, whereby the clusters move from a liquid-like state to a solidlike one. Clearly, the phase diagram for bulk helium will permit only the liquidlike phase in even the largest of clusters. Interest then rests with the possibility that superfluid clusters may be generated $^{8}$.

Tony Stace is in the School of Chemistry and Molecular Sciences, University of Sussex, Brighton BN1 9QJ, UK.

\footnotetext{
1. Sands, W. D., Bieler, C. R. \& Janda, K. C. J. chem. Phys 95, 729-734 (1991)

Hair, S. R., Cline, J. I. Bieler, C. R. \& Janda, K. C. chem. Phys. 90, 2935-2943 (1989)

3. Bieler, C. R. Evard, D. D. \& Janda, K. C. J. phys. Chem 94, 7452-7457 (1990)

4. Horn, T. R., Gerber, R. B. \& Ratner, M. A. J. chem. Phys. 91, 1812-1823 (1989)

5. Garcia-Vela, A., Villarreal, P. \& Delgado-Barrio, G. J. chem. Phys. 92, 496 (1990)

6. Goyal, S., Robinson, G. N., Schutt, D. L. \& Scoles, G. J. phys. Chem. 95, 4186-4189 (1991).

7. Hahn, M. V. \& Whetten, R. Phys. Rev. Lett. 61, 1190 1193 (1988)

8. Toennies, J. J. in Proc. CVII Summer School E. Fermi int. School Phys. (ed. Scoles, G.) 597-617 (North Holland, Amsterdam, 1990)
}

\section{New vitality}

LAST week, Daedalus proposed a 'lifecycle' for DNA. It survives the death of the organism itself (archaeological DNA millions of years old is known), and leaks back into the environment as stable drifting 'spores' of a few genes each. If such a spore happens to be present at an act of sexual fertilization, it can be incorporated into the new conception, and can start a new genetic career.

Over evolutionary time, quite a lot of spore-DNA must have managed to climb aboard the genome of each species in this way. Most of it will come from entirely different species. In its new home it will be so much meaningless parasitic junk the 'junk DNA' so mysteriously present in most creatures. But a spore from the same species will change the creature's heredity. This is mutation.

Any dead tissue, says Daedalus, can release spores of DNA: even fallen hair, skin fragments, nail clippings and so on, discarded during life. A spore from a living 'donor' might easily find its way into some unrelated act of human lovemaking. The resulting child would then not be totally the offspring of its parents. A distant third party will have made a modest contribution.

The obvious application is to genetic engineering. If spores can be identified, and their structures understood, artificial ones could be constructed from specific regions of human DNA. The full mapping of the human genome will make it easier to choose regions which code for one precisely known characteristic. Introduced into human matings, they could produce small desired mutations to order. There must be an immune response to overcome, but this cannot be an insuperable barrier.

This minor tweaking of heredity raises far fewer problems than major interventions such as AID. Many potential parents might not be too jealous of a small controlled contribution from outside. Potential donors, too, would welcome the new technology. Even today, most childless people have twinges of regret; yet if the world's population is ever to decrease to a sensible size, many more of us will have to be childless. But with spore-DNA genetic engineering, anyone will be able to contribute a small but valued part of himself to other people's children, by donating snippets of hair or tissue for spore formation. Even after his death, his tissues could influence new generations. Physicists might happily incorporate a dash of the mathematical genius of Isaac Newton into their offspring; biologists could welcome a touch of darwinian imagination, while Lenin's tomb may prove a rich source of relic DNA for parentally hopeful politicians.

DAVID JONES 